\title{
Bullins et bilharzioses en Corse
}

\section{Répartition, fréquence et biologie de «Bulinus truncatus "}

\author{
Par J.-M. DOBY, B. RAULT, S. DEBLOCK et A. CHABAUD (1)
}

\section{1) Les gîtes à bullins en Corse pendant l'été 1965}

Bulinus truncatus a sa limite nord de répartition géographique en France sur la côte méditerranéenne, semble-t-il, puisque signalé par Germain (1930) des Pyrénées-Orientales.

En Corse, cette espèce (2) a été signalée antérieurement par Germain dans le Fango notamment, et a fait plus tard l'objet de plusieurs prospections plus ou moins systématiques : E. Brumpt (1930), Buttner et Bourcart (1956 et 1957), Mars (1961) et Grétillat (1963). Celles-ci avaient montré la fréquence en relative abondance de B. truncatus dans toutes les zones côtières du pays, à l'exception de la moitié nord de la côte orientale, c'est-à-dire au nord de l'embouchure de la Chiola, l'absence de ce mollusque dans toute cette région étant attribuée par Buttner et Bourcart à la nature géologique du terrain (3).

Ces études présentent en Corse un intérêt tout particulier, tant sur le plan médical que sur le plan vétérinaire, puisque ce gastropode est l'hôte intermédiaire possible de Schistosoma haematobium, Schistosoma bovis et Paramphistomum cervi. D'ailleurs, certains des auteurs précités, ainsi que L. Brumpt et Rocca-Serra (1956), ont envisagé les possibilités d'introduction de l'infection humaine dans l'île et étudié l'épidémiologie de la bilharziose animale dont l'existence a été reconnue à plusieurs reprises dans le sud de la Corse (E. Brumpt, 1930 ; Grétillat, 1963 ; Biguet et coll., 1962, communication orale).

Nous n'avons pas jugé inutile d'y effectuer une nouvelle prospection systématique, puisque le Comité d'Experts de l'Organisation Mondiale de la Santé a appelé encore

(1) Avec la collaboration technique, par ordre alphabétique, de A. Agbavoh, O. Bain, M.-C. Desset, C. Huchet-Saguez, N. Korbendeau, G. Philippe, J.-C. Quentin et G. Rosin.

(2) Selon Mandahl-Barth (in litt.), Bulinus truncatus de Corse appartient à la même sousespèce que les exemplaires de Sardaigne, c'est-à-dire B. truncatus rivularis (Philippi).

(3) L'absence de B. truncatus, constatée par les deux auteurs dans cette zone, coïncidait en effet étrangement avec la nature schistique ou alluvionnaire de celle-ci. Germain avait cependant signalé sa présence «dans le Fango, près de Bastia (E. Requien, O. Debeau) et aux environs de Calvi (E. Caziot)... ». De même, l'existence, avant la dernière guerre, de stations à bullins au sud de Bastia, au nord de l'étang de Biguglia, nous a été signalée oralement par le Docteur Gaud. Ces stations n'ont fait l'objet d'aucune publication, à notre connaissance du moins. 


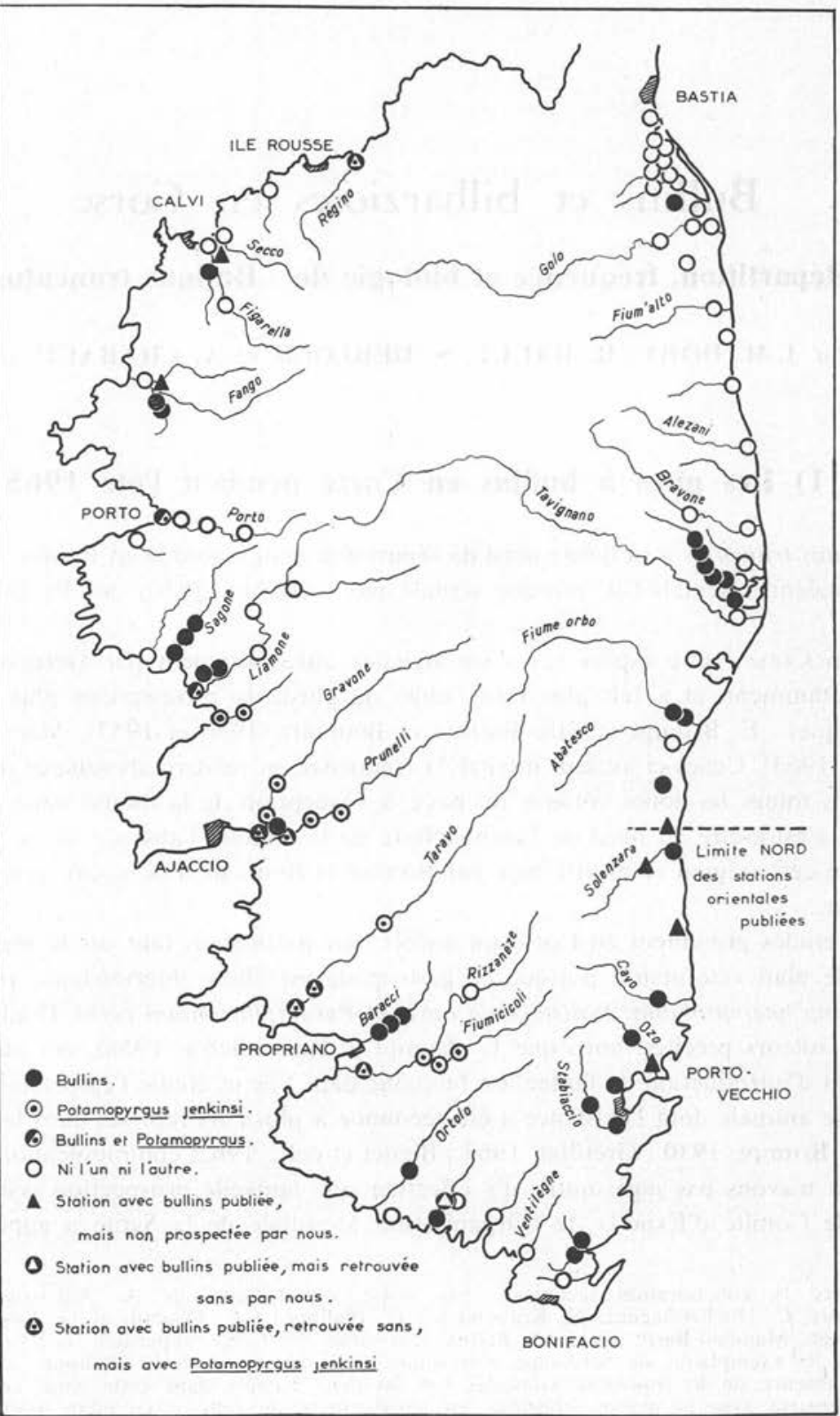


récemment l'attention * sur la nécessité d’obtenir des renseignements plus précis sur la fréquence des schistosomoses animales dans les différentes parties du monde 》 ( $3^{e}$ rapport du Comité d'Experts, $\mathrm{n}^{\circ} 299$, page 20).

Au cours de ces prospections, qui ont porté, du 15 juillet au 15 août 1965 , sur toutes les zones côtières de la Corse, à l'exception de l'extrême nord (Cap Corse et Golfe de St-Florent) ont été examinées de 250 à 300 collections d'eau diverses (mares résiduelles dans le lit des fleuves et ruisseaux, diverticules latéraux et parties à courant lent de ceux-ci, zones marécageuses, roubines et canaux de drainage ou d'irrigation, maresabreuvoirs pour le bétail dans les pâtures) ; 55 hébergeaient des bullins. Elles se situent comme suit :

(*) Grande mare-dépotoir de l'aérodrome de Bastia, au sud de l'étang de Biguglia.

${ }^{*}$ ) - Série de mares résiduelles, sur plusieurs kilomètres du lit de l'Aréna, près d'Aléria.

(*) - Petit ruisseau se jetant dans l'étang de Diane, sous le pont de la route nationale N. 198.

$\left.{ }^{*}\right)$ - Plusieurs mares résiduelles dans le lit du Fiume Orbo, à proximité du pont sous la N. 198.

${ }^{*}$ ) - Grande mare-abreuvoir sur le bord de la N. 198 près de Vix, au niveau de l'aérodrome.

- Mares résiduelles dans l'embouchure de la Solenzara et sous le pont de la N. 198.

- Le Cavo, l'Ozo (mares résiduelles au niveau des ponts sous la N. 198).

- Grandes mares-abreuvoirs sur le bord de la N. 198 à $3 \mathrm{~km}$ au nord de Porto-Vecchio.

- Le Stabiaccio (nombreuses mares résiduelles au niveau de la N. 198, et sur les bords du courant même, près du pont sous la Départementale D. 159).

$\left({ }^{*}\right)$ - Le Canalli et deux autres ruisseaux sous la N. 198 au nord de Bonifacio.

- Mare-abreuvoir au sud de Monaccia («mare de Brumpt », 1930) (4).

- L'Ortolo au niveau du pont sous la N. 196.

- Le Baracci (très nombreuses mares résiduelles sur plusieurs kilomètres depuis l'embouchure).

- Petite mare résiduelle sous un pont d'un petit affluent de la Gravone.

- Diverticule sans courant à l'embouchure du Liamone.

- Deux mares isolées à quelques centaines de mètres du Liamone, sur la rive nord.

- Nombreuses mares résiduelles sur plusieurs kilomètres dans le lit de la Sagone.

(*) - Le Porto, au niveau de l'élargissement terminal formant lagune, juste avant la mer.

$\left.{ }^{*}\right)$ - Nombreuses cuvettes rocheuses dans le lit du Colombo (affluent du Fango) au niveau de la route N. 199.

(*) - Le Piani, affluent du Figarella, au niveau de sa jonction avec celui-ci.

Il s'ajoute par conséquent plusieurs stations nouvelles (5) à la liste dressée par les

(4) L'année précédente, à la même époque, près de Monaccia, nous avions trouvé des bullins dans plusieurs petites mares résiduelles dans le lit du Spartano. Ces collections d'eau ont été retrouvées négatives cette année, les mares situées près de la route ayant entre temps subi un énergique traitement anti-anophélien à base d'hydrocarbures, ce qui est peut-être à l'origine de la disparition des bullins.

(5) Stations précédées d'un astérisque dans la liste ci-dessus. 
prospecteurs qui nous ont précédés; cette dernière coïncide sensiblement avec la nôtre, à l'exception des stations observées dans les lits des Rizzaneze, Fiumicicoli, Taravo, Prunelli, Gravone et Regino qui apparaissent présentement sans bullins.

Dans la plupart de ces stations retrouvées négatives, nous avons constaté, au contraire, l'acclimatement, le plus souvent en extrême abondance (estimation par exemple à plusieurs milliards d'individus sur une dizaine de kilomètres du Rizzaneze), d'un petit gastropode Hydrobiidé, Potamopyrgus jenkinsi (Smith 1889), qui n'avait été signalé en 1961 qu'en un point unique bien limité de la Corse.

Il est possible que ce gastropode envahissant les collections d'eau puisse, par sa pullulation, éliminer la plupart des autres mollusques pré-existants à son invasion (à l'exception de certains Ancylidés et des Néritines, que nous lui avons trouvés parfois associés).

Si cet antagonisme se vérifiait, on pourrait envisager, compte tenu de ses possibilités extraordinaires d'adaptation à des biotopes très variés [eaux douces, saumâtres, stagnantes. courantes; climats froids (Finlande) et chauds (Portugal, Espagne et Corse)], son utilisation comme moyen de contrôle biologique dans la lutte contre les mollusques hôtes-intermédiaires des affections humaines et vétérinaires par trématodes (6).

Notre étude a mis en évidence que, contrairement aux observations de Buttner et Bourcart qui n'avaient pas trouvé de bullins sur la côte orientale au nord de l'embouchure de la Chiola, il existe un certain nombre de stations dans cette zone, la plus nordique étant située à quelques kilomètres au sud de Bastia, ce qui semble invalider les conclusions que ces auteurs aient cru pouvoir tirer de l'absence du gastropode et de la nature géologique du sol.

Il convient cependant de remarquer que le nombre de stations à bullins est allé en augmentant progressivement du nord vers le sud.

Les observations écologiques effectuées pour chaque collection d'eau où figurait B. truncatus (7) démontrent qu'en ce qui concerne du moins les gîtes d'été (gîtes de maintenance en période sèche), il n'existe pas de biotope typique à bullins. Toutes les collections d'eau, mises à part les saumâtres et les très courantes, quelles que soient leur profondeur, leur superficie, la nature de leur fond et de leurs bords, leur ensoleillement, leur richesse en végétation ou en débris végétaux morts, sont susceptibles d'héberger $B$. truncatus en relative abondance (8); il peut s'agir de mares bordant les lits des rivières et torrents, en eau ou à sec, ou autour des piliers de ponts, de grandes mares permanentes dans les pâtures, etc...

Nous avons limité nos prospections aux régions côtières, nous conformant en cela aux observations de nos prédécesseurs. Cependant, nous croyons les bullins capables

(6) L'étude détaillée de la répartition actuelle de Potamopyrgus en Corse et son utilisation éventuelle pour le contrôle biologique ont fait l'objet de deux publications antérieures (Doby et coll., 1965).

(7) Cette étude détaillée fera l'objet d'une autre publication (Doby et coll., 1966).

(8) La mare-dépotoir de l'aérodrome de Bastia, grande dépression inondée à quelques centaines de mètres de celui-ci (cf. photo), est un bel exemple de tolérance à une polution relativement importante de l'eau; de même, la mare-abreuvoir de Monaccia où les bullins, également très abondants, vivaient dans une eau par endroits recouverte d'une couche flottante de bouses de bovidés de plusieurs centimètres d'épaisseur. 
de remonter assez haut en amont dans le lit de certains torrents, puisque nous en avons retrouvés dans le Stabiaccio, sur les bords du courant même, assez loin à l'intérieur des terres, au niveau du pont, sous la D. 159. De même, nous les avons retrouvés en extrême abondance (un adulte environ par $2 \mathrm{~cm}^{2}$ par endroit), avec d'innombrables pontes, à une altitude de $210 \mathrm{~m}$, dans des vasques rocheuses du lit du Columbo.

\section{2) Recherche sur les bilharzioses}

Notre étude sur la répartition géographique et la biologie des bullins pendant l'été 1965 a été complétée par la recherche des formes larvaires des deux schistosomes dont cette espèce est l'hôte intermédiaire, Schistosoma bovis et Schistosoma haematobium.

Tous les bullins récoltés ont été mis en observation pendant 24 heures environ, en tubes à essais exposés au soleil, pour la recherche de l'émission spontanée des furcocercaires, puis ils furent écrasés un à un sous la loupe binoculaire stéréoscopique pour la recherche des sporocystes et des cercaires immatures; 3.500 bullins furent ainsi examinés.

Sur les 55 points d'eau hébergeant $B$. truncatus, un seul contenait des mollusques de cette espèce présentant des furcocercaires de schistosomes (9). La station, fort localisée, se situe à quelques kilomètres de Propriano, sur la côte ouest, dans le lit du Baracci, partiellement à $\mathrm{sec}$ en été à cette période ; elle se réduisait alors à une unique petite mare. Une dizaine d'autres gîtes simultanément prospectés dans un rayon de quelques centaines de mètres de part et d'autre du même endroit (mares-abreuvoirs, fossés, diverticules secondaires du courant principal), tous fort riches en bullins, étaient négatifs (683 mollusques examinés dont 482 sujets moyens et gros).

Le biotope était constitué, lors du prélèvement, par un creux résiduel sous une souche d'arbre, de $2 \mathrm{~m}$ sur $1 \mathrm{~m}$ environ de surface, d'une profondeur maximale de $0,30 \mathrm{~m}$. L'eau en était trouble, dépourvue d'algues et de végétation, mais fort riche en débris végétaux (branches et brindilles mortes). Le fond était vaseux et boueux, l'ensoleillement relativement important. Les bullins étaient particulièrement abondants, de toutes tailles, et les pontes nombreuses.

Etaient associées aux bullins, mais nettement moins abondantes, les espèces suivantes : Limnaea peregra (Müll.), Gyraulus laevis (Alder), Hippeutis complanatus (L.) et Ancylus costulatus Küster (10).

Les 109 bullins du gîte examinés un par un révélèrent 26 sujets infectés par des furcocercaires ( 0 sur 17 petits, 7 sur 46 moyens et 19 sur 46 gros) ; 5 de ces 26 présentaient une association de furcocercaires de schistosomes et de cercaires de Paramphistomum. Ce second trématode parasitait en effet 55 des 109 bullins examinés.

(9) Plusieurs espèces de cercaires et de métacercaires de genres différents ont été aussi mises en évidence, avec une fréquence variable en fonction des collections d'eau étudiées. II s'agit notamment de larves d'Echinostomidés et de Paramphistomidés (Deblok et coll., 1966).

(10) Nous remercions vivement notre collègue M. le Professeur Mandahl-Barth qui a bien voulu déterminer ces mollusques. Une étude écologique détaillée, en association avec lui, fera l'objet d'une note ultérieure. 
TABLeaU I

Etude comparative des différentes furcocercaires

(mensurations en microns)

\begin{tabular}{|c|c|c|c|}
\hline & $\begin{array}{c}\text { Furcocercaires } \\
\text { du Baracci }\end{array}$ & $\begin{array}{c}\text { Schistosoma } \\
\text { haematobium (1) }\end{array}$ & $\begin{array}{l}\text { Schistosoma } \\
\text { bovis (2) }\end{array}$ \\
\hline Corps $\ldots \ldots \ldots \ldots$ & $\begin{array}{l}140 \times 48 \\
(120-160)(45-50) \\
(3)\end{array}$ & $\begin{array}{c}158 \times 53 \\
(120-180)(48-65)\end{array}$ & $\begin{array}{c}153 \times 42 \\
(110-180)(29-59)\end{array}$ \\
\hline Organe céphalique ... & 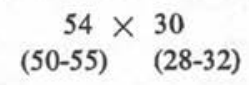 & $\begin{array}{c}62 \\
(40-70)\end{array} \times \frac{38}{(32-40)}$ & (4) \\
\hline Tronc caudal... . & $\begin{array}{c}195 \\
(175-210)(25-30)\end{array}$ & $\begin{array}{c}207 \times 32 \\
(160-234)(32-37)\end{array}$ & $\begin{array}{c}213 \times 30 \\
(202-228)(18-37)\end{array}$ \\
\hline Fourchons $\ldots \ldots \ldots$ & $\begin{array}{c}77 \\
(65-90)\end{array}$ & $\begin{array}{c}86 \\
(75-93)\end{array}$ & $\begin{array}{c}70 \\
(40-88)\end{array}$ \\
\hline Ventouse ventrale ... & $\begin{array}{c}18 \\
(17-19)\end{array}$ & $\begin{array}{c}23 \\
(19-24)\end{array}$ & 20 \\
\hline
\end{tabular}

(1) in CAPRON et coll. 1965.

(2) in LENGY, 1962.

(3) les chiffres inférieurs correspondent aux mensurations extrêmes.

(4) pas de mensurations données.

Les furcocercaires furent examinées d'abord à frais, puis après fixation à l'alcool à $70^{\circ}$ et coloration, ainsi que sur coupes histologiques sériées de mollusques parasités.

Les caractères suivants permettent d'affirmer leur appartenance aux schistosominés et au genre Schistosoma (11).

Ce sont des cercaires apharyngées, non ocellées, brévifourchées (longueur des fourchons inférieure à la moitié de la longueur du tronc caudal), à petite ventouse ventrale à lumière épineuse, à ventouse orale modifiée en organe céphalique de pénétration, et possédant enfin cinq paires de glandes. Les fourchons sont dépourvus de crête.

Les coupes histologiques de mollusques parasités montrent que les furcocercaires prennent naissance dans des sporocystes à paroi mince situés dans l'hépatopancréas et la glande sexuelle hermaphrodite.

La position du genre étant définie, il n'est guère possible, avec la seule morpho-

(11) Bien que, d'après Dutt et Srivastava (1952), certaines furcocercaires du genre Ornithobilharzia (cas de $O$. dattai) ne puissent pas se distinguer de celles du genre Schistosoma; $O$. dattai évolue chez un mollusque indou du genre Limnaea et, adulte, parasite les bovidés. 
logie, de dire à quelle espèce de schistosome nous avons affiaire. Le choix se trouve néanmoins limité, du fait de la nature de l'hôte, à Schistosoma bovis et éventuellement à Schistosoma haematobium.

Nous donnons dans le tableau ci-contre (tableau I) les mensurations comparées des furcocercaires que nous avons observées et de celles de Schistosoma haematobium et Schistosoma bovis relevées dans les publications les plus documentées les concernant (Capron et coll. 1965, pour la première espèce, et Lengy 1962, pour la seconde). Ce tableau ne permet pas de mettre en évidence de différences significatives entre les trois furcocercaires, surtout si l'on tient compte des variations de dimensions inhérentes aux techniques de fixation utilisées (12).

Pour Lengy, d'ailleurs, « la cercaire mature de Schistosoma bovis est, dans l'état actuel de nos connaissances, indistinguable de celle des autres espèces de schistosomes ».

Des raisons épidémiologiques nous donnent à penser qu'il s'agit de Schistosoma bovis:

$1^{\circ}$ Schistosoma haematobium n'a jamais encore été signalé de Corse à ce jour, à notre connaissance du moins.

$2^{\circ}$ Schistosoma bovis existe sur le pourtour du bassin méditerranéen et a été à plusieurs reprises signalé de Corse.

$3^{\circ}$ Du bétail pâturait dans le lit du Baracci et dans les prairies avoisinantes.

D'après notre prospection, qui a couvert la quasi totalité de la Corse, du moins dans ses zones côtières, il apparaît que l'infestation animale à Schistosoma bovis est actuellement peu répandue et, peut-être même, en régression. La station reconnue par Brumpt en 1930 à Monaccia, toujours fréquentée par le bétail, semble s'être récemment négativée, d'une façon sans doute spontanée. Encore positive en 1962 (Biguet et coll. : $1 \%$ de bullins parasités, communication orale), nous l'avons trouvée négative en 1965 (750 bullins examinés). Grétillat, travaillant sensiblement dans la même région, n'avait décelé en 1963 aucun schistosome dans 270 mollusques examinés.

Ceci se confirme encore par l'absence d'œufs de Schistosoma bovis dans une centaine de matières fécales de bovidés récoltées au hasard à proximité de la mare de Monaccia et à proximité du point d'eau trouvé positif dans le lit du Baracci. Pourtant, l'examen microscopique de ces matières fécales a consisté en un examen direct suivi de la mise en jeu de la technique de concentration de Janeckso et Urbanyi, si efficace pour la diagnose des affections par trématodes.

D'ailleurs, les services vétérinaires corses considèrent comme d'importance tout à fait secondaire et de simple intérêt théorique dans l'île la présence chez les bovidés de Schistosoma bovis (13).

(12) Une idée de l'importance de ces différences selon les auteurs peut être obtenue par la lecture du tableau comparatif concernant Schistosoma haematobium figurant dans la publication de Capron et coll.

(13) Nous remercions vivement M. le Docteur vétérinaire A. Romani, Directeur des Services vétérinaires de la Corse, qui a accepté de nous recevoir à ce sujet et de nous faire part des renseignements en sa possession concernant cette affection. 
Pourtant, dans d'autres pays méditerranéens, la bilharziose animale peut parfois revêtir une importance considérable. En Espagne par exemple (Botija 1959), un foyer de schistosomose ovine s'est révélé en 1953, dans un troupeau de 1.240 têtes. 600 animaux plus particulièrement atteints sur 800 touchés durent être abattus.

Compte tenu de la réceptivité expérimentale des petits mammifères à Schistosoma bovis (souris, hérissons, cobayes, E. Brumpt, 1930 et 1949, gerbilles, Arfaa et coll., 1965), nous avons jugé intéressant de rechercher les schistosomes chez ces derniers. Ainsi, d'une part, les schistosomes adultes ont été systématiquement recherchés dans les vaisseaux mésentériques et hépatiques de 220 petits mammifères sauvages appartenant à six espèces différentes (tableau II) capturés pendant les étés 1964 et 1965 dans

\section{TABLEAU II}

Recherche de bilharziose bovine chez les petits mammifères en Corse 1964 et 1965

\begin{tabular}{|c|c|c|c|c|c|c|}
\hline Origine & $\begin{array}{l}\text { Rattus } \\
\text { rattus } \\
\text { alex- } \\
\text { andrinus }\end{array}$ & $\begin{array}{c}\text { Mus } \\
\text { musculus } \\
\text { brevi- } \\
\text { rostris }\end{array}$ & $\begin{array}{c}\text { Apodemus } \\
\text { sylvaticus } \\
\text { dichrurus }\end{array}$ & $\begin{array}{l}\text { Mustela } \\
\text { minuta } \\
\text { corsicana }\end{array}$ & $\begin{array}{c}\text { Crocidura } \\
\text { russula } \\
\text { cyrnensis }\end{array}$ & $\begin{array}{l}\text { Erinaceus } \\
\text { europaeus }\end{array}$ \\
\hline Sagone $\ldots \ldots \ldots$ & $13(0)(1)$ & $35 \quad(0)$ & $11(0)$ & $1 \quad(0)$ & - & $1 \quad(1)$ \\
\hline Talone-Aléria $\quad \therefore$ & $4 \quad(4)$ & $19(12)$ & 3 (1) & 一 & - & - \\
\hline Porto-Vecchio .. & $3 \quad(3)$ & $6 \quad(6)$ & - & 一 & 一 & - \\
\hline Monaccia (2) ... & $5 \quad(4)$ & $57(27)$ & 7 (4) & $1 \quad(1)$ & $2(0)$ & 一 \\
\hline Bicchisano.... & $4 \quad(4)$ & 1 (1) & 31 (24) & 一 & $1 \quad(0)$ & - \\
\hline Porticcio-Ajaccio & $5 \quad(4)$ & $9 \quad(3)$ & $1 \quad(0)$ & - & 一 & 一 \\
\hline Total : 220 (99). & 34 (19) & $127(49)$ & $53(29)$ & 2 (1) & $3 \quad(0)$ & $1 \quad(1)$ \\
\hline
\end{tabular}

(1) entre les parenthèses le nombre des animaux examinés également immunologiquement.

(2) en partie capturés autour de la mare «Brumpt » elle-même.

la zone de bilharziose bovine reconnue, ou bien là où des bullins ont été observés en abondance. D'autre part, la recherche immunologique de la bilharziose dans le sérum d'un certain nombre de ces petits mammifères (99) a été pratiquée (14). Toutes ces recherches ont été négatives.

(14) Examens par technique de précipitation en gélose (Ouchterlony et immuno-électrophorèse), effectués au Laboratoire de Parasitologie de la Facultế de Médecine de Lille (Professeur J. Biguet) par M. le Professeur agrégé A. Capron, à qui nous renouvelons ici nos vifs remercie ments pour sa collaboration. 
En ce qui concerne la bilharziose humaine, c'est le problème de son éventuelle importation qu'il convient d'envisager. Jusqu'à maintenant, en effet, à notre connaissance du moins, aucun cas autochtone de bilharziose vésicale humaine n'a été publié de Corse (15).

La possibilité de l'établissement de cette affection dans l'île a pourtant été évoquée à plusieurs reprises (L. Brumpt et Rocca-Serra, 1956) ; le risque siège dans l'introduction éventuelle de sujets porteurs en provenance de régions où la bilharziose sévit à l'état endémique. Les conclusions relativement optimistes de Buttner et Bourcart en 1957 se justifiaient alors par des probabilités minimes de réalisation du cycle épidémiologique: pas ou peu d'immigration de porteurs et gîtes à mollusques trop « sauvages » pour s'offrir facilement au contact du réservoir de virus d'abord et de l'individu sain ensuite.

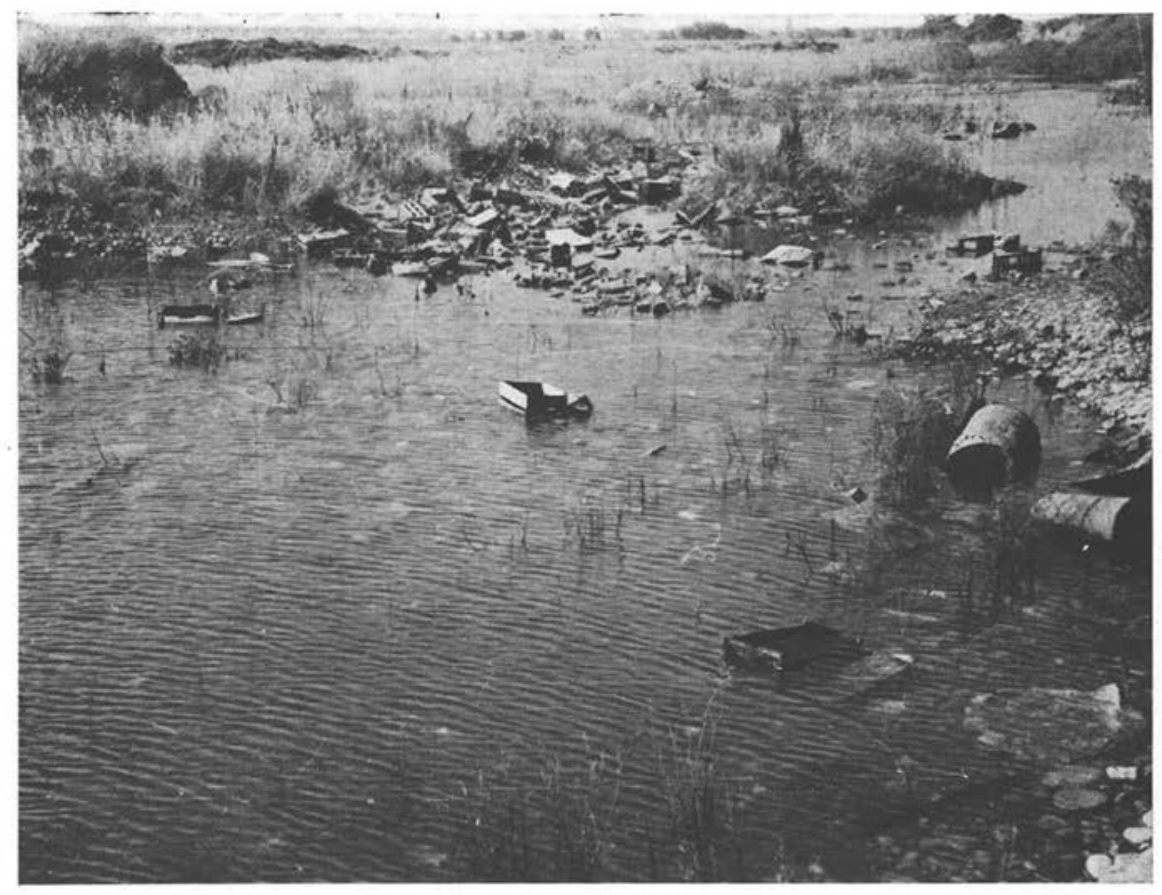

Pното $\mathrm{N}^{\circ}$ 1. - Mare-dépotoir de l'aérodrome de Bastia

(15) Nous avons eu cependant connaissance d'un cas de bilharziose vésicale humaine, qui aurait été vérifié par la présence d'œufs avec éperon terminal dans les urines, chez un sujet ayant séjourné aux environs d'Ajaccio et n'ayant par ailleurs jamais voyagé dans d'autres régions susceptibles de présenter de la bilharziose. Ce diagnostic fut effectué avant la dernière guerre à l'Hôpital maritime de Lorient et nous a été rapporté par M. le Docteur Carn de Quimper, ancien médecin colonial lui-même, que nous remercions particulièrement ici. Malheureusement, en dépit de nos recherches, des renseignements complémentaires sur ce cas n'ont pu être retrouvés en raison de la destruction des archives de cet Hôpital à la fin des dernières hostilités. 


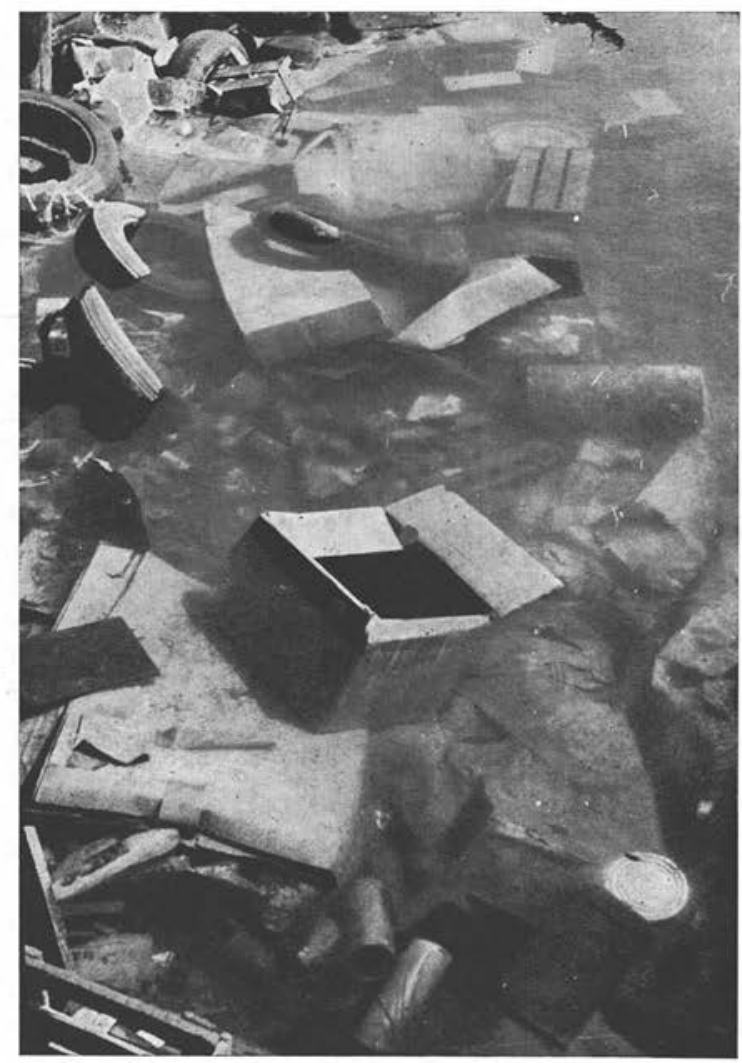

Рното $\mathrm{N}^{\circ}$ 2. - Détail du gîte de la photo précédente. Sur les cartonnages immergés sont visibles des points noirs. $\mathrm{Ce}$ sont des bullins. De nombreuses pontes peuvent être là récoltées

Nos conclusions personnelles sont sensiblement plus pessimistes. Si la plupart des gîtes, essentiellement ruraux, ne sont fréquentés que par les troupeaux, un certain nombre cependant se situent à portée de l'homme, rural ou citadin.

Or, les circonstances politiques, jointes à «la course au soleil » lors des vacances, contribuent actuellement à modifier grandement et rapidement le visage traditionnel de ce département. Les exploitations agricoles modernes utilisent l'eau de certaines collections permanentes pour les besoins de leurs cultures. Les ouvriers de ces exploitations ne dédaignent pas ces collections d'eau à l'occasion, pour la toilette ou la baignade en fin de journée. La main-d'œuvre saisonnière s'importe principalement d'Afrique du Nord; elle s'importera peut-être à l'avenir d'Afrique Noire. De l'une et l'autre origine, elle pourra contaminer les bullins et être ainsi le point de départ de 


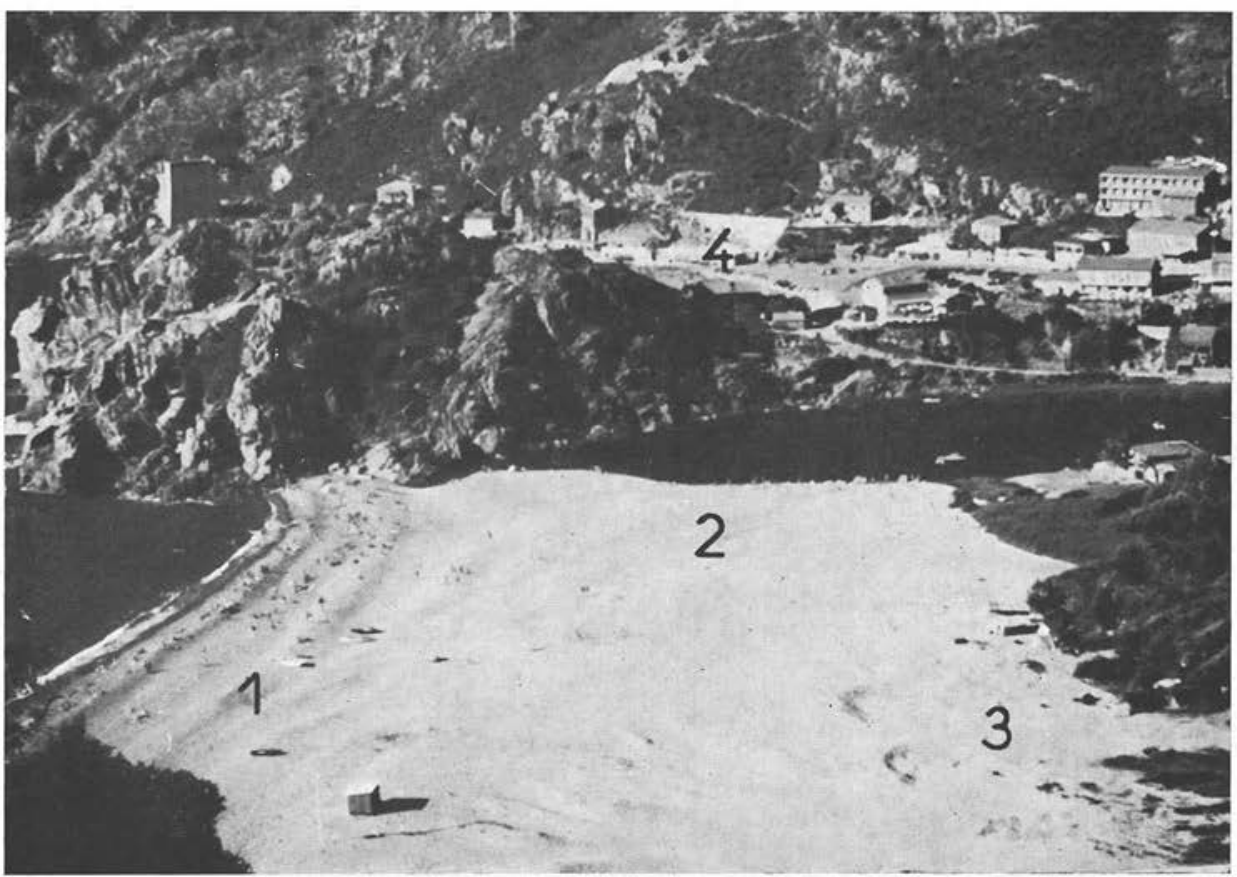

Рното $\mathrm{N}^{\circ}$ 3. - Porto. Plan d'eau aménagé sur le bord de mer dans la portion terminale du fleuve Porto: 1) plage du bord de mer ; 2) plage d'eau douce sur le plan d'eau avec de nombreux baigneurs. Sur tout le pourtour de ce plan d'eau, y compris les lieux de baignade, peuvent être récoltés des bullins par milliers; 3) sous les arbres, terrain de camping aménagé; 4) chantiers de constructions immobilières avec main-d'œuvre nord-africaine

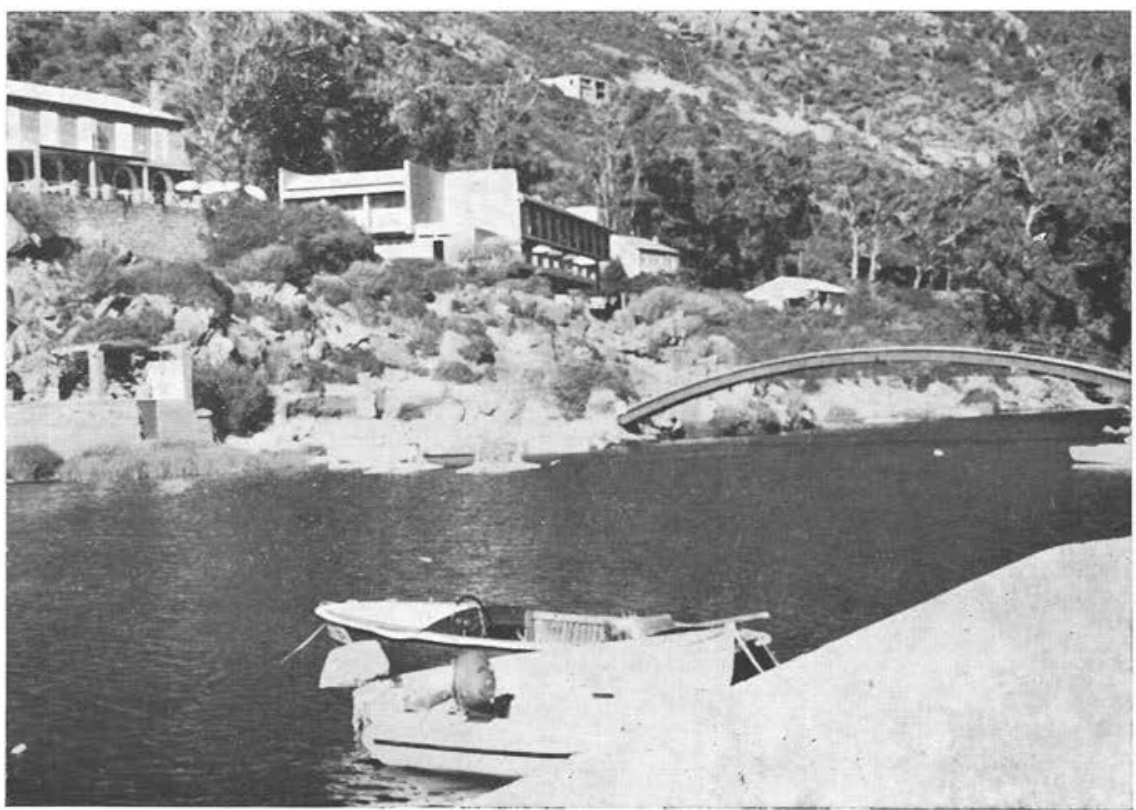

Рното $\mathrm{N}^{\circ}$ 4. - Détail du site de la photo précédente 
petites épidémies saisonnières. Ce danger n'est pas absolument imaginaire. Un agriculteur de la région de Bastia, qui utilisait pour l'irrigation de ses terres l'eau de la grande mare de l'aérodrome où pullulent les bullins, ne nous a-t-il pas confié qu'au moins un de ses ouvriers, d'un groupe de Marocains en provenance de la région de Tanger, quelques mois auparavant, était un «pisseur de sang».

Nous avons eu par ailleurs sous nos yeux des exemples d'ouvriers agricoles importés se baignant dans des vasques d'eau aménagées dans le lit de rivières où se trouvaient des bullins en abondance.

La mise en valeur du sol s'accompagne d'un essor de la construction immobilière, génératrice, elle aussi, d'un appel de main-d'œuvre nord-africaine. L'attrait du soleil, qui fait surgir les chantiers de résidences nouvelles, entraîne de plus la naissance estivale de nombreux terrains de camping dont les emplacements se situent de préférence sous les ombrages des lits de rivières partiellement asséchées en été ou à leurs abords immédiats. Les points d'eau permanents, souvent riches en bullins, tentent les vacanciers ou leurs enfants pour la baignade, les ablutions ou les besoins domestiques.

Le plus grand danger serait réalisé par la conjonction de ces divers phénomènes. Celle-ci n'est absolument pas utopique. Un bon exemple peut être donné par l'extraordinaire site de l'embouchure du Porto, avec des chantiers immobiliers dominant la lagune d'eau douce toute proche du rivage de mer, et un immense terrain de camping; le plan d'eau est très fréquenté par tout un peuple de vacanciers et de campeurs auxquels viennent se joindre les ouvriers des chantiers voisins lors de leur période de repos (16). Or, la lagune pullule de bullins (17).

Le premier cas certain de bilharziose humaine autochtone n'est donc pas inconcevable. La Corse réunit dès à présent, et sans doute encore pour plusieurs années, en été du moins, l'ensemble des conditions requises pour réaliser à l'occasion la chaîne épidémiologique assez inhabituelle pour la France: œufs de schistosomes, bullins, homme,

\section{Bibliographie}

Arfaa (F.), Sabbaghian (H.) et Bijan (H.), 1965. - Studies on Schistosoma bovis in Iran. Trans. Roy. Soc. Trop. Med. a. Hyg., 59, 681.

BotiJa (R.-S.), 1955. - Sur la présence de la schistosomiase ovine en Espagne. Bull. Off. internat. des Epizooties, 43, 433.

(16) Dès septembre 1965, à notre retour, nous avons demandé à la Direction départementale de la Santé de la Corse des renseignements concernant l'apport de main-d'œuvre étrangère en Corse au cours de ces dernières années. Nos demandes sont malheureusement restées à ce jour sans réponse.

(17) Toutefois, nous avons trouvé là, associés aux bullins, des Potamopyrgus jenkinsi, mais en très petit nombre et sur une étendue limitée, ce qui semble traduire une introduction récente dans ce biotope. Les observations faites en d'autres points de Corse (Doby et coll., 1965) ,comme il a été dit plus haut, nous amènent à penser qu'il y a là peut-être un facteur épidémiologique défavorable à l'implantation de la bilharziose en ce point. 
Brumpt (E.), 1930. - Cycle évolutif complet de Schistosoma bovis. Infection naturelle en Corse et infection expérimentale de Bullinus contortus. Ann. Parasit. hum. et comp., 8, 17.

—, 1949. - Précis de Parasitologie, Masson éd., Paris.

Brumpt (L.-C.) et Rocca-Serra (J.-P. DE), 1956. - Danger de l'introduction en Corse de nouvelles souches de paludisme et de la bilharziose vésicale. Bull. Acad. Nat. Med., $\mathrm{n}^{\text {os }} 21,22$ et 23,425 .

Buttner (A.) et Bourcart (N.), 1956. - Mission épidémiologique en Corse (sept.). Rapport au Ministère de la Santé (Paris) et à la Préfecture de la Corse (Ajaccio) (document dactylographié).

—, —, 1957. — Etude des facteurs épidémiologiques qui président à la création d'un foyer de bilharziose humaine. Observations faites au Brésil et en Corse. Bull. Soc. Path. exot., 50, 473.

Capron (A.), Deblock (S.), Biguet (J.), Clay (A.), Adenis (L.) et Vernes (A.), 1965. Contribution à l'étude expérimentale de la bilharziose à Schistosoma haematobium. Bull. Organ. Mond. Santé, 32, 755.

Deblock (S.), Doby (J. M.), Сhabaud (A.) et Rault (B.), 1966. - Formes larvaires de trématodes parasites de Bulinus truncatus en Corse. Ann. Parasit. hum. et comp., 41 , en préparation.

Doby (J. M.), Chabaud (A.), Mandahl-Barth (G.), Rault (B.) et Chevallier (H.), 1965 Extension en Corse du Mollusque gastropode Potamopyrgus jenkinsi (Smith, 1889) (Hydrobiidae). Bull. Muséum Hist. Nat., 37, 833.

-, Mandahl-Barth (G.), Chabaud (A.), et Deblock (S.), 1965. - Elimination de Bulinus truncatus de collections d'eau connues pour l'héberger par Potamopyrgus jenkinsi (Smith, 1889) (Hydrobiidés) et utilisation éventuelle de ce mollusque pour le contrôle biologique des bilharzioses. C.R. Acad. Sci., 261, 4244.

-, - Rault (B.) et AgBavoh (A.), 1966. - Observations écologiques sur Bulinus truncatus (Gastropode) en Corse dans ses gîtes de saison sèche. Bull. Soc. Zool. France, en préparation.

Dutt (S.) et Srivastava (H.), 1952. - On the morphology and life history of a new mammalian blood-fluke, Ornithobilharzia dattai n. sp. (preliminary report). Parasitol., $42,144$.

Germain (L.), 1930. - Mollusques terrestres et fluviatiles. Faune de France, Paris, Lechevalier édr.

GrÉTILlAT (S.), 1963. - Epidémiologie de certaines affections à trématodes des animaux domestiques en Corse (Bilharziose bovine et distomatose bovine et ovine). Ann. Parasit. hum. et comp., 38, 471.

LENGY (J.), 1962. - Studies on Schistosoma bovis (Sonsino, 1876) in Israël. I. Larval stages from egg to cercaria. Bull. Res. Counc. of Israël, 10, 1.

MARS (P.), 1961. - Recherches sur quelques étangs du littoral méditerranéen français et sur leurs faunes malacologiques. Thèse Doctorat ès-Sciences, Paris.

(Laboratoire de Parasitologie et Zoologie appliquée de la Faculté de Médecine de Rennes) 